\title{
Dye-dope Polystyrene-coated Gold Nanorods: Towards Wavelength Tuneable SPASER
}

\author{
Peng Gu, David J S Birch, Yu Chen* \\ Photophysics Group, Centre for Molecular Nanometrology, Department of Physics, Scottish \\ Universities Physics Alliance, University of Strathclyde, 107 Rottenrow, Glasgow G4 0NG, UK \\ Email: y.chen@strath.ac.uk
}

\begin{abstract}
We present the synthesis and photophysical study of a new type of fluorescent gold core-polystyrene shell nanoparticles fabricated by emulsion polymerization at neutral $\mathrm{pH}$. The thickness of the PS shell can be controlled by varying the synthesis conditions. Decrease in the fluorescence intensity and lifetime of Rhodamine 800 (Rh800) were observed, indicating energy transfer from $\mathrm{Rh} 800$ to gold nanorods. This study suggests the possibility of exploiting dye-doped polystyrene shells as a gain media to compensate the energy loss of longitudinal surface plasmon resonance of gold nanorods and paving the way for eventually realizing a SPASER (Surface Plasmon Amplification by Stimulated Emission of Radiation) optical source of tuneable wavelength.
\end{abstract}

\section{Introduction}

The possibility of fabricating a SPASER (Surface Plasmon Amplification by Stimulated Emission of Radiation) source has attracted considerable attentions during the last 10 years, because of its potential applications from sensing and biomedicine to imaging and information technology[1-6]. The SPASER concept was first proposed by Stockman and Bergman in 2003[7] and can be described as where the gain media near a noble metal core transfers energy to a resonant surface plasmon (SP) mode of the metal core. The SP stimulates further transitions in the gain medium, leading to the excitation of more identical SPs in the same SP mode, driving the action of the SPASER[8-10]. Under this mechanism, a SPASER based nanolaser was demonstrated in 2009 using a core-shell structure consisting of a gold spherical core and dye-doped silica shell that completely compensates the energy loss of localized surface plasmon and realizes a SPASER process[11].
Localized surface plasmon resonance (LSPR) represents a collective of coherent charge density oscillations confined to metal nanoparticles and metallic nanostructures, whose resonance wavelength is sensitive to permittivity changes of the environment, as well as to the size and shape of the nanoparticle[12-14]. Compared to other nanocrystals, gold nanorods (GNRs) offer significant advantage as their LSPR properties can be tailored by synthetically tuning their sizes and shapes. There are two plasmon modes in GNRs: the longitudinal mode (L mode) associated with the electron oscillations along the length axis, and the transverse mode ( $\mathrm{T}$ mode) excited by light polarized along the transverse direction of the nanorod[15]. The L mode is sensitive to the shape (aspect ratio) of the particle and can be synthetically tuned[16-18] across a broad spectral range coving the visible and nearinfrared regions. A GNR based SPASER thus has advantages of wavelength tunability and polarization sensitivity[19,20]. In addition, the threshold of a nanorod based SPASER can be much lower than that of a sphere based 
structure[21], as the imaginary part of the dielectric function of gold varies with wavelength and has a minimum near $700 \mathrm{~nm}[22]$.

To this end we have investigated the synthesis of dye-doped GNRs core shell structures. Rhodamine 800 (Rh800) was chosen because of its absorption and emission near 700 $\mathrm{nm}$. Silica coating of gold nanoparticles can be obtained with a good degree of control using the Stöber method[23]. However, the extreme alkaline or acidic growth environment required by this method can limit the choice of dyes; Rh800 for example suffers a dramatic decrease in fluorescence intensity at $\mathrm{pH}>10$. Here we report the synthesis and photophysical study of a new type of fluorescent core/shell nanoparticles through emulsion polymerization. A modified polystyrene (PS) coating method is applied to grow GNR core-PS shell particles doped with Rh800 at neutral pH[24]. The thickness of the PS shell can be controlled by varying the synthesis conditions. Static optical spectroscopy studies revealed stable dye doped core/shell nanoparticles in water solution. Dynamic fluorescence spectroscopic measurements revealed strong energy coupling between gold cores and the surrounding Rh800, indicating energy loss compensation of surface plasmon resonance.

\section{Methods}

\subsection{Synthesis of GNR}

GNRs were synthesized using a seeded growth method[25]. To prepare the seed solution, a CTAB solution $(7.5 \mathrm{ml}, 0.2 \mathrm{M})$ was mixed with $2.5 \mathrm{ml}$ of $0.001 \mathrm{M} \mathrm{HAuCl}_{4}$ and the mixture was stirred for $10 \mathrm{~min} .0 .6 \mathrm{ml}$ of ice-cold $0.01 \mathrm{M}$ $\mathrm{NaBH}_{4}$ was added to the mixture, and slowly stirred for another $2 \mathrm{~min}$. The solution was kept in room temperature for at least 3 hours before usage. To prepare a growth solution, $2.971 \mathrm{~g}$ of BDAC was first mixed with CTAB solution (50 ml, 0.12-0.2 $\mathrm{M}$ depending on the aspect ratio). After 10-minute's stirring, $50 \mathrm{ml}$ of 0.001 $\mathrm{M} \mathrm{HAuCl}_{4}$ was added under vigorous stirring for
$7 \mathrm{~min}$. To this solution, $0.004 \mathrm{M} \mathrm{AgNO}_{3}(0.5-2$ $\mathrm{ml}$, depending on the aspect ratio) was added, and after 5-minute's gentle stirring, $700 \mu \mathrm{l}$ of $0.0778 \mathrm{M}$ ascorbic acid was added and stirred for $5 \mathrm{~min}$. Finally, $80 \mu \mathrm{l}$ seeds were slowly added to the growth solution. The solution was kept at room temperature for rod growth.

\subsection{PS coating}

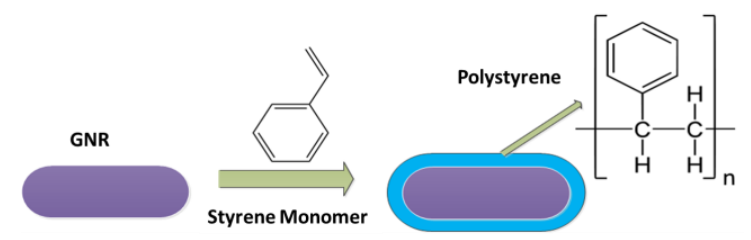

FIGURE 1 Scheme of Polystyrene coating

A simple synthesis method[24] was used to coat GNRs with PS, as schematically shown in Figure 1. To grow PS shells of different thickness, 4, 6, 8,10 , and $12 \mu \mathrm{l}$ of styrene monomer were added to $10 \mathrm{ml}$ GNRs solutions (OD=1, sample 1 to 5), respectively. After stirring for $5 \mathrm{~min}, 16,24,32$, 40 , and $48 \mu 1$ of $0.1 \mathrm{M}$ solutions of the initiator ammonium persulphate (APS) were added to above solutions and kept stirring for $15 \mathrm{~min}$. The PS-coated GNRs were centrifuged (12000 r/min $30 \mathrm{~min}$ ) and re-suspended in $10 \mathrm{ml}$ distilled water after growth for 2 hours, 1 day, 2 days, and 1 week.

To dope dye into the coating, $4 \mu \mathrm{l}$ of styrene monomer and $1 \mathrm{mg}$ of $\mathrm{Rh} 800$ were added to 10 $\mathrm{ml}$ of the GNRs solution (OD=1). The solution was stirred for $20 \mathrm{~min}$ and $16 \mu \mathrm{l}$ of a $0.1 \mathrm{M}$ APS was added. The polymerization was allowed to take place at room temperature. The solution was stirred for 20 min then sat for 1 hour. The Rh800doped PS-GNRs were centrifuged and washed twice (12000 r/min, 30min) and re-suspended in distilled water for characterization.

\subsection{Optical characterisation}

UV-vis spectra were measured using Jasco V-600, ranging from $200 \mathrm{~nm}$ to $900 \mathrm{~nm}$. HORIBA Jobin Yvon's Fluorolog-3 Spectrofluorometer was used for static fluorescence measurements. Time- 
correlated sing-photon counting (TCSPC) spectroscopy was performed using a HORIBA Jobin Yvon IBH 5000U fluorescence lifetime system. Non-linear least squares impulse reconvolution analysis of fluorescence decay curves was performed using the IBH DAS 6 software.

\section{Results and discussion}

\subsection{PS coated GNRs}
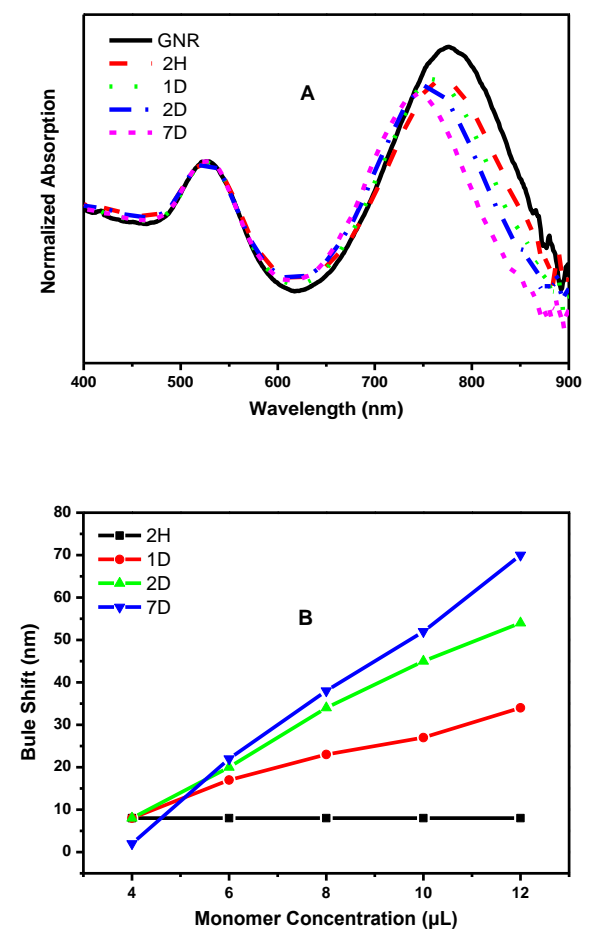

FIGURE 2 (A) Normalized UV-vis extinction spectra of PS-coated GNRs with monomer concentration of $12 \mu \mathrm{l}$ after growth for 2 hours, 1 day, 2 days, 1 week, and original GNRs (all normalized against $\mathrm{T}$ modes); (B) the blue shift of $\mathrm{L}$ modes as a function of time from PS-coated GRNs grown with $4,6,8,10$, and $12 \mu \mathrm{l}$ of monomer.

Figure 2A shows extinction spectra of GNRs with PS shells grown in 2 hours, 1 day, 2 days and 1 week with a monomer concentration of 12 $\mu 1$. The effect of the coating on the optical response of GNRs is apparent in comparison to that of original GNRs. While the wavelength of the $\mathrm{T}$ mode remains almost unaltered, the $\mathrm{L}$ mode shifts to shorter wavelengths due to a decrease in the local permittivity around the gold core introduced by the PS shell. The increased blue shifts at longer growth time suggest the formation of thicker coating layers. Figure 2B compares the blue shift of 5 samples of different monomer concentration $(4,6,8,10$, and $12 \mu \mathrm{l})$ after growth for 2 hours, 1 day, 2 days, and 1 week. No difference was found among the five samples after 2 hours' growth, even if an excess amount of monomer and APS initiator exists in the solutions. However, the difference becomes obvious after 1 day's growth, where samples of higher monomer concentration have larger blue shifts. This suggests that the thickness of PS shell can be controlled by varying monomer concentration and growth time.

The growth of PS layer on GNRs is through an emulsion polymerization process. It is likely that hydrophobic styrene monomer diffuses into the CTAB surfactant bilayer on the gold surface and forms a $\mathrm{CTAB} /$ styrene mixture structure on GNR. Arrival of the free APS molecules triggers polymerization of monomers inside this structure and initiates the growth of polystyrene shell. At the same time, monomer droplets in the solution supply monomers which diffuse through the aqueous phase to facilitate the further polymerization and growth of the PS layer[26,27].

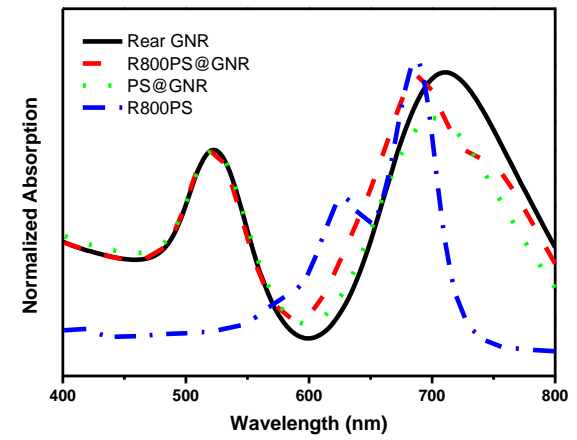

FIGURE 3 Normalized UV-vis extinction spectra of original GNRs (black), PS-coated GNRs (green), Rh800-doped PS GNRs (red), (all 
normalized against $\mathrm{T}$ modes) and $\mathrm{Rh} 800$-doped PS sphere (blue).

\subsection{Rh800 doped PS-coated GNRs}

Figure 3 shows the normalized UV-vis extinction spectra of original GNRs, PS-coated GNRs, Rh800-doped PS GNRs, and R800 doped PS spheres as a control sample. A $10 \mathrm{~nm}$ blue shift was found from PS-coated GNRs, consistent with what found in Figure 2. The extinction spectrum of Rh800-doped PS GNRs shows an absorption peak at $690 \mathrm{~nm}$ sitting on a broad absorption band (L mode of GNRs). The 690nm peak resembles the main absorption of Rh800, indicating a successful dye doping.

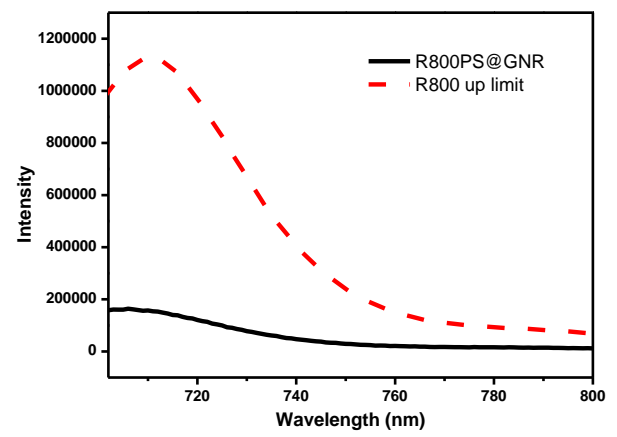

FIGURE 4 Emission spectra of Rh800-dopped PS GNRs (black) and Rh800 of calculated uplimit concentration in water solution (red).

Figure 4 shows the emission spectra of Rh800doped PS GNRs in comparison with that from a Rh800 solution $\left(3.17 \times 10^{-6} \mathrm{M}\right.$ in $10 \mathrm{ml}$ water solution). This molar concentration of the dye molecules was estimated by the known initial dye concentration in the reaction taking away the free dye molecules remaining in the solution after the reaction (concentration of dye in supernatant after centrifugation). This calculation provides the uplimit concentration of Rh800 doped in PS shells. It is clear that the fluorescence intensity of dye doped PS-GNRs is about an order of magnitude lower than that from free dye in solution. Furthermore, dynamic fluorescence spectroscopic studies compare fluorescence decay process of Rh800 in PS-GNR particles and $\mathrm{Rh} 800$ in PS spheres, as shown in figure 5. The decay curve of Rh800 in PS spheres can be fitted with a single exponential function, generating a lifetime of $0.71 \mathrm{~ns}$. Two exponential fitting of the decay curve of Rh800 in PS-GNRs revealed a first lifetime component of $0.71 \mathrm{~ns}(85.8 \%)$ and second lifetime component of $0.1 \mathrm{~ns}$ (14.2\%). While the first lifetime is consistent with that of $\mathrm{Rh} 800$ in PS spheres, the second lifetime is much shortened. Both decreased fluorescence intensity and lifetime suggest an energy coupling between dye and GNRs.
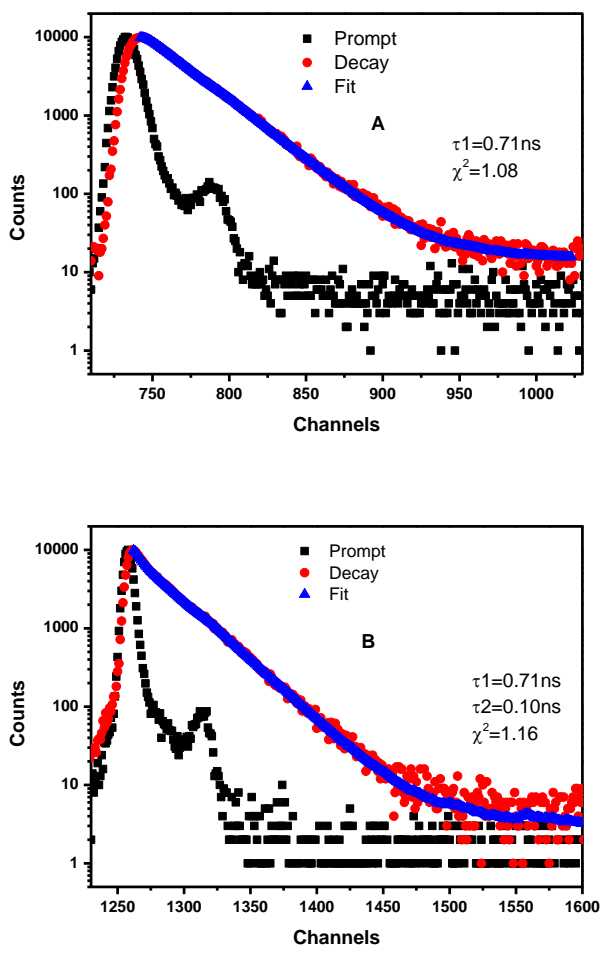

FIGURE 5 Fluorescence decay curves of (A) Rh800-doped PS sphere and (B) Rh800-doped PS GNRs.

\subsection{Influence of surface plasmon on energy transfer}

Energy transfer from dye to gold nanoparticles has been described using a surface energy transfer (SET) model, where the distance dependence of the transfer rate scales at $4^{\text {th }}$ power[28]. Previous studies have found that this 
energy transfer is enhanced when a surface plasmon mode overlaps with the excitation wavelength[29,30]. In order to study the surface plasmon effect on the energy transfer between Rh800 and GNRs, longer GNRs with an L mode at $783 \mathrm{~nm}$ were used. The extinction spectrum of Rh800 doped PS-GNRs-L, Figure 6, shows a shoulder on the short wavelength side of the surface plasmon mode which matches the main absorption peak of Rh800 in a PS environment. Figure 7A shows decreased fluorescence intensity from Rh800-doped PS-GNRs-L by about an order of magnitude, compared to the Rh800 reference sample $\left(5.39 \times 10^{-6} \mathrm{~mol}\right.$ in 10 $\mathrm{ml}$ water solution). A shorter lifetime component of $0.28 \mathrm{~ns}(4.2 \%)$ was observed along with a lifetime of $0.73 \mathrm{~ns}(95.8 \%)$ for the Rh800-doped PS-GNRs-L particles, as shown in Figure 7B. The existence of a shorter lifetime component together with decreased fluorescence intensity indicates an energy transfer from dye to GNR. Compared to GNRs with a longitudinal SP of $720 \mathrm{~nm}$, the red shift of SP from long GNRs reduced the spectral overlapping between the absorption of dye and SP, resulting in a weakened energy transfer process.

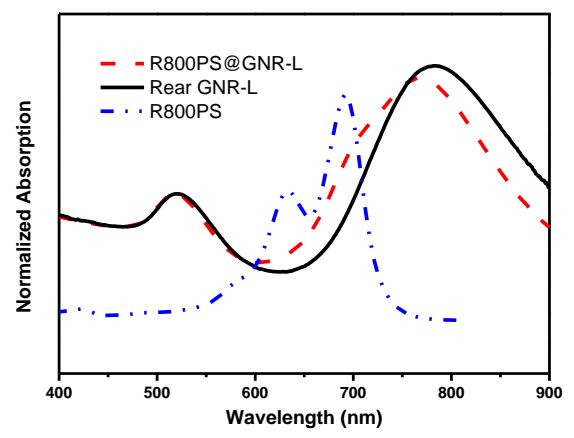

FIGURE 6 Normalized UV-vis extinction spectra of original GNRs (black), Rh800-doped PS GNRs-L (red), (all normalized against transverse modes) and Rh800 doped PS sphere (blue).

\section{Conclusions}

In summary, we have successfully synthesized a new type of fluorescence GNR core-PS shell nanoparticles via emulsion polymerization at neutral $\mathrm{pH}$. The thickness of the PS shell can be controlled by varying monomer concentration and growth time. Decrease in the fluorescence intensity and lifetime of Rh800 were observed in comparison to that from dye doped PS spheres, indicating an energy transfer from Rh800 to GNRs. Moreover, this energy transfer is enhanced when the SP overlaps with the absorption and emission of Rh800. This energy transfer compensates for the energy loss of surface plasmon and thus this study brings further insight to creating nanorod-based wavelength tuneable SPASERs.
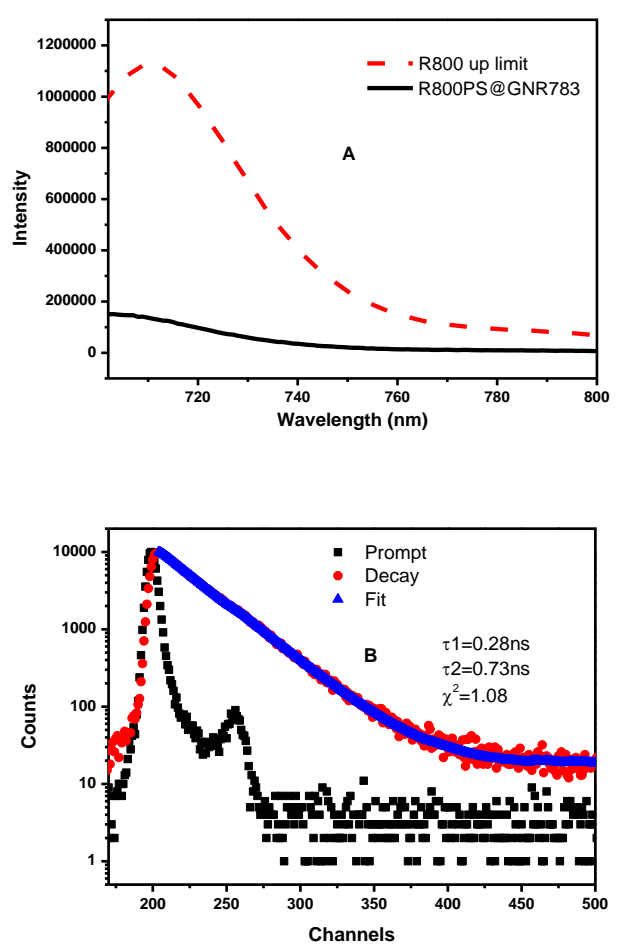

FIGURE 7 (A) Emission spectra of Rh800dopped PS GNRs-L (black) and Rh800 of calculated uplimit concentration in water solution (red) and (B) fluorescence decay curve of Rh800doped PS GNR-L.

\section{Acknowledgement}

We wish to thank the EPSRC, BBSRC and SFC for their financial support. PG also wishes to thank RSC and IOP for their travel grants. 


\section{References}

[1] Stockman M I 2008 Spasers explained Nat. Photonics 2 327-9

[2] Zhang H, Zhou J, Zou W and He M 2012 Surface plasmon amplification characteristics of an active three-layer nanoshell-based spaser J. Appl. Phys. 112 074309

[3] Stockman M I 2011 Nanoplasmonics : The physics behind the applications Phys. Today 39-44

[4] De Luca A, Grzelczak M P, PastorizaSantos I, Liz-Marzán L M, La Deda M, Striccoli M and Strangi G 2011 Dispersed and encapsulated gain medium in plasmonic nanoparticles: a multipronged approach to mitigate optical losses. ACS Nano 5 5823-9

[5] Zheludev N I, Prosvirnin S L, Papasimakis N and Fedotov V a. 2008 Lasing spaser Nat. Photonics 2 351-4

[6] Flynn R A, Kim C S, Vurgaftman I, Kim M, Meyer J R, Mäkinen a J, Bussmann K, Cheng L, Choa F-S and Long J P 2011 A room-temperature semiconductor spaser operating near $15 \mu \mathrm{m}$ Opt. Express 19 8954

[7] Bergman D and Stockman M 2003 Surface Plasmon Amplification by Stimulated Emission of Radiation: Quantum Generation of Coherent Surface Plasmons in Nanosystems Phys. Rev. Lett. $901-4$

[8] Stockman M 2011 Spaser Action, Loss Compensation, and Stability in Plasmonic Systems with Gain Phys. Rev. Lett. 106 14

[9] Stockman M I 2011 Loss compensation by gain and spasing. Philos. Trans. A. Math. Phys. Eng. Sci. 369 3510-24

[10] Stockman M I 2011 Nanoplasmonics: past, present, and glimpse into future $O p t$. Express 1922029

[11] Noginov M A, Zhu G, Belgrave A M, Bakker R, Shalaev V M, Narimanov E E, Stout S, Herz E, Suteewong T and Wiesner U 2009 Demonstration of a spaser-based nanolaser. Nature $\mathbf{4 6 0} 1110$ 2

[12] Zheng J, Zhou C, Yu M and Liu J 2012 Different sized luminescent gold nanoparticles. Nanoscale 4 4073-83
[13] Zhang Y, Yu J, Birch D J S and Chen Y 2010 Gold nanorods for fluorescence lifetime imaging in biology. J. Biomed. Opt. 1520504

[14] Chen Y, Preece J A and Palmer R E 2008 Processing and characterization of gold nanoparticles for use in plasmon probe spectroscopy and microscopy of biosystems. Ann. N. Y. Acad. Sci. 1130 201-6

[15] Perezjuste J, Pastorisantos I, Lizmarzan L and Mulvaney P 2005 Gold nanorods: Synthesis, characterization and applications Coord. Chem. Rev. 249 1870-901

[16] Orendorff C J and Murphy C J 2006 Quantitation of metal content in the silverassisted growth of gold nanorods. J. Phys. Chem. B 110 3990-4

[17] Jana N R, Gearheart L and Murphy C J 2001 Wet Chemical Synthesis of High Aspect Ratio Cylindrical Gold Nanorods $J$. Phys. Chem. B 105 4065-7

[18] Jana N R, Gearheart L and Murphy C J 2001 Seed-Mediated Growth Approach for Shape-Controlled Synthesis of Spheroidal and Rod-like Gold Nanoparticles Using a Surfactant Template Adv. Mater. 13 1389-93

[19] Zhang Y, Yu J, David B J S, Chen Y 2011 Gold nanorods for applications in biological imaging, Proc. SPIE 7910, 79101H

[20] Meng X, Kildishev A V, Fujita K, Tanaka K and Shalaev V M 2013 Wavelengthtunable spasing in the visible. Nano Lett. 13 4106-12

[21] Liu S-Y, Li J, Zhou F, Gan L and Li Z-Y 2011 Efficient surface plasmon amplification from gain-assisted gold nanorods. Opt. Lett. 36 1296-8

[22] Johnson P B and Christy R W 1972 Optical Constants of the Noble Metals Phys. Rev. B 6 4370-9

Pastoriza-Santos I, Pérez-Juste J and LizMarzán L M 2006 Silica-Coating and Hydrophobation of CTAB-Stabilized Gold Nanorods Chem. Mater. 18 2465-7

Obare S O, Jana N R and Murphy C J 2001 Preparation of Polystyrene- and Silica-Coated Gold Nanorods and Their Use as Templates for the Synthesis of Hollow Nanotubes Nano Lett. 1 601-3 
[25] Liu M and Guyot-Sionnest P 2005

Mechanism of silver(I)-assisted growth of gold nanorods and bipyramids. $J$. Phys.

Chem. B 109 22192-200

[26] Cochin D, Laschewsky A and Nallet F 1997 Emulsion polymerization of styrene using conventional, polymerizable, and polymeric surfactants. A comparative study Macromolecules 9297 2278-87

[27] Ravve A 2012 Principles of Polymer Chemistry vol 31 (New York, NY: Springer New York)

[28] Yun C S, Javier A, Jennings T, Fisher M, Hira S, Peterson S, Hopkins B, Reich N O and Strouse G F 2005 Nanometal surface energy transfer in optical rulers, breaking the FRET barrier. J. Am. Chem. Soc. 127 3115-9

[29] Zhang Y, Birch D J S and Chen Y 2011 Two-photon excited surface plasmon enhanced energy transfer between DAPI and gold nanoparticles: Opportunities in intra-cellular imaging and sensing Appl. Phys. Lett. 99103701

[30] Racknor C, Singh M R, Zhang Y, Birch D J S and Chen Y, 2013, Energy transfer between biological labelling dye and gold nanorods, 2013, Methods Appl. Fluoresc. in press 